\title{
UNEQUALLY POWERED CRYPTOGRAPHY WITH PHYSICAL UNCLONABLE FUNCTIONS FOR NETWORKS OF INTERNET OF THINGS TERMINALS
}

\author{
Bertrand Cambou \\ School of Informatics Computing and Cyber Systems \\ Northern Arizona University ; Building 90 Room 110 \\ Flagstaff, AZ86011, USA \\ Bertrand.cambou@nau.edu
}

\begin{abstract}
In this paper, cryptographic architectures based on unequal computing resources are modelled, and experimentally verified with Window-10 PCs powered with Intel I-7 processor. Low power IoT terminal devices generate keys from physical unclonable functions, which can be recognized by the server through a matching engine. We are here proposing methods to modulate the efficiency of the matching engine by injecting erratic bits in the keys, and/or by fragmenting the keys in smaller sub-keys. When the environment is hostile, the difficulty in matching the keys can be adjusted in such a way that the server needs access to increasingly powerful computing power to recognize the terminal device thereby placing attackers with inferior computing power at a strong disadvantage. The objective of this research work is to find, through the modelling effort, the conditions where unequally powered cryptography can take advantage of high performance computers to protect networks of IoT terminals.
\end{abstract}

Keywords: IoT security, network security, physical functions.

\section{INTRODUCTION}

Most encryption schemes assume that the computing power needed by the communicating parties is balanced between them; decryption usually requires the same power as encryption, which is for example presented by Yin (2015). The security of networks with the internet of things (IoT) terminals can be difficult, due to the heterogeneous nature of such networks. IoTs are often powered by small microcontrollers, which are unable to handle demanding cryptographic schemes. Physical unclonable functions (PUFs) can act as the fingerprint of the hardware. They can simplify the key distribution scheme to a network of IoT, as described by Herder, Yu, and Koushanfar (2014), as well as by Holcomb, Burleson, and $\mathrm{Fu}$ (2008). However, the finger prints of the PUFs vary as they age, and due to temperature or other parameter drifts, which require error correcting methods, as suggested by Rahman, Rahman, Forte, and Tehranipoor (2016). In most encryption schemes a single bit mismatch in the fingerprints is not acceptable, therefore the importance of error correction is very high. Cryptographic schemes injecting noise based on random number generators to confuse the adversary, and increase entropy, have also the potential to protect networks of IoTs without burdening the computing power of the noise generating party. Several important publications in this area are noticeable, such as the international patent application of Fiske (2016), which describes how to hide a public key exchange in noise, the US patent application of Janus, Martin, and Savagaonkar (2017), which presents how to prevent patent recognition in stored information by injecting noise, and the US patent of Fernando, Wang, and Soysa (2008), which use noise injection to enhance security.

This paper presents an architecture exploiting the computing power available at the server level, to recognize networks of IoTs, and their PUFs, in a selectively noisy environment. Only servers equipped with 
high powered computing elements will then be able to handle noisy signals, thereby preventing eavesdropping with less powerful computers.

In section 2, the background section, PUFs based cryptographic protocols are described. The conventional method to detect and correct the drifts of the fingerprints of the PUFs is presented, as well as novel schemes that replace this conventional method with search engines at the server level such as response based cryptography (RBC). PUFs and RBCs are essentials to the work presented in this paper. In section 3, models for the unequally powered cryptography are proposed. The asymmetry between server and IoT can be increased by incrementally injecting noise at the terminal level. Conversely, the asymmetry can be reduced by fragmenting the cryptographic keys. Models to predict the respective effect of noise injection, and key fragmentation are presented, to optimize the protocols, and reduce false rejection rates (FRR). The objective of section 4 is to present the experimental analysis validating the models presented above. With commercial SRAM based PUFs, and a PC, the efficiency of the unequally powered scheme is characterized under various levels of noise, and key fragmentation. Finally, in Section 5, the modeling tool developed in this work is used to anticipate the conditions in which unequally computing cryptography can leverage the computing power of HPCs to protect networks of IoTs.

\section{BACKGROUND}

\subsection{Cryptography with physical unclonable functions}

Physical unclonable functions (PUFs) can be used as cryptographic primitives for authentication, and to generate keys for encryption schemes. PUFs are designed with electronic components, for example memory devices, which can exploit the unique micro-variations created during fabrication. PUFs can be generated effectively by Static RAM memories, as presented by Chen, Willem, Maes, Sluis, and Selimis (2017), Maes, Tuyls, Verbauwhede (2009), by Resistive RAM, Chen (2015), Cambou and Orlowski (2016), Cambou, Afghah, Sonderegger, Taggart, Barnaby, and Kozicki (2017), by Flash memories, Prabbu, Akel, Grupp, Yu, Suh, Kan, and Swanson (2011), or other Nanomaterials, Gao, Ranasinghe, Al-Sarawi, Kavehei, and Abbott (2016). When embedded in client devices, PUFs act as the digital "fingerprints" of the hardware. The initial "fingerprints", also called challenges, are stored as references in a server during enrollment cycles, while the fresh "fingerprints" of the PUFs, called responses, are queried at each authentication cycles. When the responses match the challenges with only small percentages of erratic bits, the authentications are positive. Due to parametric drifts, temperature variations, and aging, the streams of bits of the responses could differ by $5-20 \%$ from the streams of bits of the challenges stored by the server during the enrollment process. In order to be able to use the PUF challenge-response-pairs (CRP), as cryptographic keys, error correcting methods are applied. A single bit mismatch between the challenges, and the corrected responses is not acceptable, therefore highly effective the error correcting methods are needed. Examples of correcting methods are the ones presented by Becker, Wild, and Güneysu (2015), Boehm (2010), Delvaux, Schellekens, and Verbauwhede (2015), Korenda, Afghah, and Cambou (2018), and Taniguchi, Shiozaki, Kubo, and Fujino. These methods consume a lot of power, which is prohibitive for some networks of IoTs, and can weaken the security of the entire protocol, as presented by Hiller, Weiner, Rodrigues, Birkner, and Sigl (2013), as well as Kang, Hori, Katashita, Hagiwara, Iwamura (2014).

Conversely, methods using ternary states do not need heavy correction, and can potentially reduce the computing power needed by the IoTs. Shown in Fig.1, is an example of the cryptographic system based on PUFs. During enrollment the PUF challenges are stored in a look up table of the server. A ternary representation of the challenge differentiates the cells with predictable state, 0 or 1 , from the cells with a fuzzy and unstable state, called X. Only these predictable cells are then kept for challenge-response-pair matching, to reduce the error rates. Such a protocol is described by Cambou, and Telesca (2018), and by Cambou, Flikkema, Palmer, Telesca, and Philabaum (2018). To initiate the cryptographic key generation, the server sends the handshake containing a random number $\boldsymbol{T}$, a mask $\boldsymbol{M}$, and the Helper. The random number $\boldsymbol{T}$, together with a password, generated through the hash function message digests that 


\section{Cambou}

independently point at a particular address in the look up table, and in the PUF array. The mask $\boldsymbol{M}$ transmits to the client, the position of the fuzzy cells that are rejected to reduce error rates. The Helper, a data stream of various length, feeds the error correcting engine to generate error-free responses. Error correction schemes, which can consume significant computing power, are potential liabilities when the device is in hostile territory.

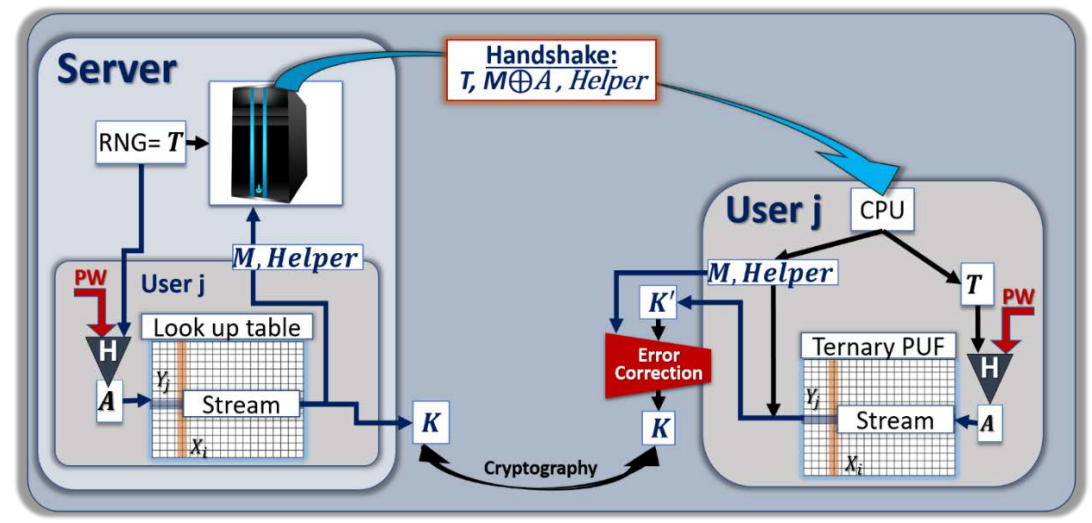

Figure 1: Example of cryptographic protocol with ternary PUF and error correcting scheme.

\subsection{Response based cryptography}

As the protocol described in section 2.1, can be called "challenge-based cryptography", a "response-based cryptography" (RBC), uses un-corrected responses from a constellation of PUFs securing client devices. An example of cryptographic protocol protected by RBC is shown in Fig.2, and is presented by Cambou, Philabaum, Booher, and Telesca (accepted paper for March 2019). As done with the challenge-based cryptography, the secure server sends the handshake to the client device to allow the independent generation of the cryptographic key from the PUF, however the helper is not needed anymore. In this protocol the key generated by the client device from the PUF is used to encrypt a known authentication message such as the user ID, with the advanced encryption standard (AES). The RBC engine independently encrypts the same message with the key generated from the look up table, and compares it with the cipher received from the client. If the two do not match, the RBC matching engine creates new keys with Hamming distance of one from the original key, which are also used to encrypt the authentication message, and compare the ciphers. The process iterates with greater Hamming distances to the point that a match is uncovered. In case of a negative matching, before the maximum acceptable Hamming distance, the authentication is rejected.

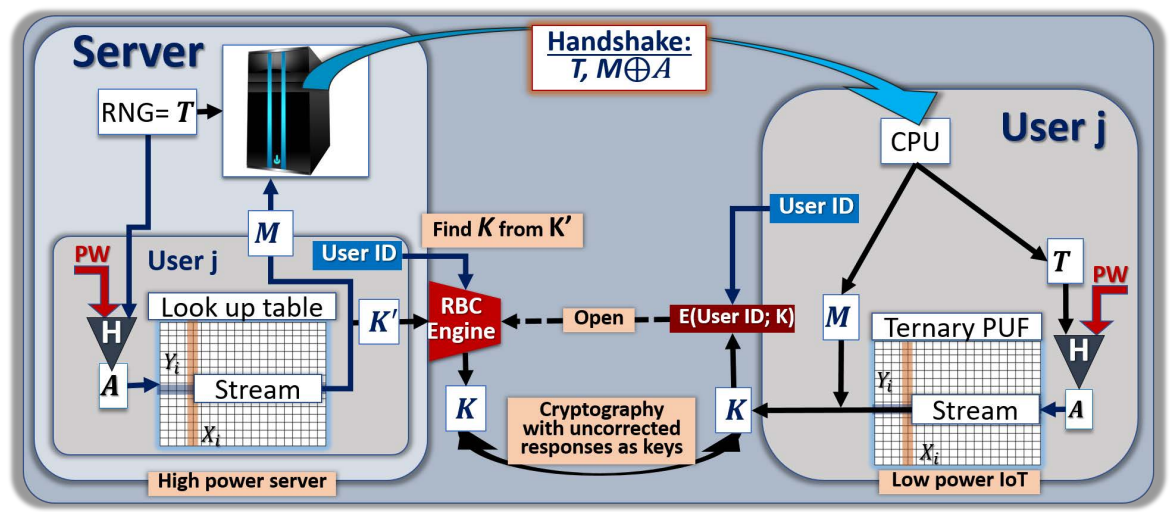

Figure 2: Example of cryptographic protocols with ternary PUFs and RBC.

The scheme is unequal, regarding the need of computing power: the client devices can use less powerful microcontrollers, and smaller memory components; at the server level, the RBC engines consume great 
amounts of computing power when the Hamming distances challenge-response are high. The elimination of the helpers simplifies the communication between server and clients, and the latency at the client device level is reduced, which enhances security.

\section{MODELS FOR UNEQUALLY POWERED CRYPTOGRAPHY}

\subsection{General description}

As reported in prior work, the use of noise injection adds entropy to the ciphers exchanged through insecure channels, and improves the encryption schemes. The objective is to create large asymmetries in the need of computing power between server and client devices by injecting noise at various levels. This noise injection can be selectively increased, to prevent malicious parties pretending to be the legitimate server, during attacks such as man-in-the-middle. The objective of unequally powered cryptography is to control the asymmetry between server, and client device. The level of power inequality should be an adjustable parameter that can be increased when the client devices are exposed to hostile environments. In section 3, two conflicting parameters are modeled, i) the level of mismatch between the fresh responses, and the one stored in the server that can increase through noise injection, and ii) the level of fragmentation of the keys generated from the responses, which reduce asymmetry. In the experimental section, the model is validated with a Window-10 PC powered with Intel I-7 processor. The model is used to extrapolate the efficiency of an HPC with 2,600 cores, which we intend to validate in future work. The block diagram of the proposed architecture is shown in Fig.3.

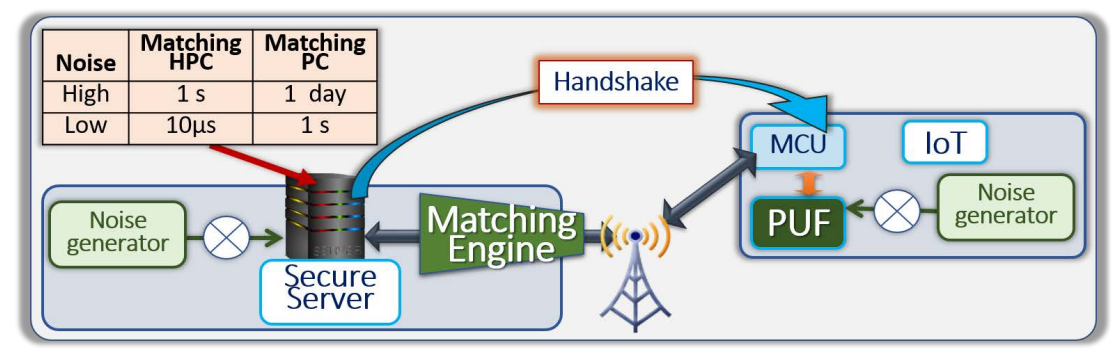

Figure 3: Block diagram of unequally powered cryptographic protocols with ternary PUFs.

This architecture is based on the use of ternary PUFs, and RBC, as they are presented in the background section $\S 2$. Two important changes are however, noise injection and key fragmentation:

The design of the architecture is based on the following:

$>$ The noise injection either at the client level, or server level, makes the keys generated by the PUF responses after noise injection, different from the ones generated by the challenges stored in the look up table. As a result the computing resources needed to establish a secure communication with the RBC increases with higher noise levels. At low level, a PC is powerful enough to find a matching key in seconds, but needs an extended period of time at a high level of noise. Conversely our model is showing that high performance computers (HPC) need only seconds to establish the communication. Large institutions such as governments, or big corporations own high performance computers (HPC) while the number of hackers with HPC is small, and often known by intelligence agencies. Conversely, the noise injection can be reduced when the client device is on safe ground, for example in a secure facility. The reduction lowers the cost associated with the $\mathrm{RBC}$ matching algorithm, allowing regular servers to interact with the client devices.

$>$ Fragmentation of the keys. The Response based cryptographic (RBC) scheme presented above in section 2, is good enough to support secure communications with ternary PUFs, however such a scheme does not support noise injection greater than 5 bits for 256-bit keys. Section 3-3 describes, and models a method that fragments the keys to increase the capability of the RBC to more rapidly recognize keys 


\section{Cambou}

with higher levels of noise. This allows the injection of much higher numbers of erratic bits, which further increases the inequality between the server and client device, in terms of computing power.

\subsection{Modelling the effect of the noise}

Fig. 4 presents the modelling of the effect of noise injection on the computing resources needed by the RBC matching engine. The length of the challenge-response-pairs is 256 bits. When " $a$ " bits are injected in the responses by the noise generator, the number of possible 256-bit streams Ns having a Hamming distance " $a$ " from the challenges stored in the look up table is given by:

$$
\mathrm{Ns}=\left(\begin{array}{c}
256 \\
a
\end{array}\right) \text {. }
$$

The server needs to independently encrypt each of these streams, with AES in this case, and compare them with the cipher sent by the client device. Fig.4 (see page 6) shows the result of the model estimating the time needed to process all streams when " $\boldsymbol{a}$ " increases from $\boldsymbol{a}=0$ to $\boldsymbol{a}=8$, and to find a matching stream with RBC. The model, in accordance with experimental results, assumes that each AES cycle $\tau_{\mathrm{o}}$ takes $1 \mu$ s on the PC, which is multiplied by Ns-a at a given Hamming distance " $a$ ". For example the equation to find the average matching time $\tau$ with Hamming distance of 4 is given by:

$$
\tau=\tau_{\mathrm{o}}+\tau_{\mathrm{o}}\left(\begin{array}{c}
256 \\
1
\end{array}\right)+\tau_{\mathrm{o}}\left(\begin{array}{c}
256 \\
2
\end{array}\right)+\tau_{\mathrm{o}}\left(\begin{array}{c}
256 \\
3
\end{array}\right)+\tau_{\mathrm{o}}\left(\begin{array}{c}
256 \\
4
\end{array}\right) / 2 .
$$

This matching time is lowered with parallel computing; in this model we assumed $60 \%$ efficiency for the HPC which is realistic considering the highly repetitive tasks to encrypt with AES a large number of 256bit keys. For example, when 6 bad bits are injected by the noise generator, it takes only a few seconds for the HPC to authenticate the client device, and weeks for a regular PC, which is largely unequal. If the client puts a time limit to a successful enrolment of one minute, both PCs, and regular servers are excluded from the protocol. When the noise generator injects only 3 bad bits, PCs and regular servers can respond in less than one minute. This noise can be injected by the client devices, or by the server.

Noise injection by the client devices. In Fig. 3 each client device has a noise generator embedded with the PUF. After key generation, " $\boldsymbol{a}$ " bits randomly selected in the keys are replaced by their opposite $(0 \rightarrow 1$; $1 \rightarrow 0$ ) to force an error. The PUFs also naturally generate random bit errors, which could create false rejection rates (FRR). The SRAM PUF without elimination of the unstable cells is showing about 3-5\%

error rates, this drops to $10^{-5}$ after 1,000 enrollment cycles of the SRAM and the masking of the unstable cells. The method suggested here is to mask the unstable cells, and inject noise in a PUF known to have defect rates in the $10^{-5}$ range. For example, as shown by the model of Fig.4, when 5 bits are injected in 256bit long keys, the RBC engine with HPC needs $400 \mathrm{~ms}$ to find the matching key, versus 20 days for a single core Window-10 PC. Only 2 bits can be injected in the PUF when the server is powered by a PC. This method of noise injection has limited applicability, it does not work well with PUFs having higher error rates. The algorithms based on the fragmentation of the keys, as presented in section 3.3, allows the use of naturally erratic PUFs, and can reduce the false rejection rates.

Noise injection by the server. The injection of erratic bits can be driven from the server by modifying the mask sent to the client devices during handshake. With the masking of the fuzzy states, the expected challenge-response-pair error rate is almost zero, without making the error rates are much higher, about $3.5 \%$ in the case of a commercial SRAM. A partial masking can therefore modulate the error rate, and act as a noise injector. Such methods to inject noise, were experimentally verified. Other examples include, but not limited by, the use of noisy electronics in the emission, transmission, and reception of the information needed by the key exchange. These noises can be filtered, or amplified to modify the noise injection.

\subsection{Modelling the effect of the fragmentation of the responses with RBC}

The efficiency of the RBC, as presented in section 2 with 256-bit keys, reaches its limitation when the error rate is too high, even powerful HPCs are unable to perform the validation of the key exchange in a reasonable time. For example the model presented above suggests that it will take an HPC five hours to 


\section{Cambou}

confirm an 8-bit mismatch, which is not acceptable for most applications. The objective of the algorithm fragmenting the responses is to largely increase the efficiency of the matching engine of the RBC, and to be able to inject more noise, for example 40 bad bits per response, which takes $\mathbf{2 . 5} \mathbf{1 0}^{\mathbf{3 2}}$ times longer than 8 bad bits per response, as shown eq(3):

$$
\mathrm{Na}-8=\left(\begin{array}{c}
256 \\
8
\end{array}\right)=410^{14} ; \mathrm{Na}_{-40}=\left(\begin{array}{c}
256 \\
40
\end{array}\right)=110^{47} \rightarrow \mathrm{Na}-40 / \mathrm{Na}-8=2.510^{32}
$$

The proposed method is to fragment the 256-bit keys generated with PUF responses into two 128-bit subkeys, four 64-bit sub-keys, eight 32-bit sub-keys, 16 16-bit sub-key, or 32 8-bit sub-keys. The sub-keys are complemented with known data streams that are shared with the server during the handshake, to form 256bit sub-keys. The 256-bit sub-keys are then used to encrypt an authentication message with methods such as the advanced encryption standard (AES). The ciphers are transmitted to the RBC of the server, which finally validates the key exchange. The model presented below shows that the matching of several sub-keys is significantly faster than the matching with RBC of a single key, because for a given defect density, the average error rates of the sub-keys are proportional to the level of fragmentation of the original key.

\subsubsection{Modelling the fragmentation in two 128-bit long sub-keys of the 256-bit keys}

The matching times at various error rates " $\boldsymbol{a}$ " of the model of the fragmentation in two 128-bit long subkeys are summarized in Fig.5. The efficiency of the RBC engine is then significantly faster.

\begin{tabular}{|c|c|c|c|c|c|}
\hline Hamming " $a$ " & $\begin{array}{c}\text { Numbers of } \\
\text { 256-bit streams }\end{array}$ & $\begin{array}{c}\text { Matching } \\
\text { time PC }\end{array}$ & $\begin{array}{l}\text { Matching } \\
\text { time server }\end{array}$ & $\begin{array}{l}\text { Matc } \\
\text { time }\end{array}$ & $\begin{array}{l}\text { ching } \\
\text { HPC }\end{array}$ \\
\hline 0 & $\left(\begin{array}{c}256 \\
0\end{array}\right)=1$ & $5 \mu \mathrm{s}$ & $50 \mathrm{~ns}$ & 0.05 & ns \\
\hline 1 & $\left(\begin{array}{c}256 \\
1\end{array}\right)=256$ & $1.2 \mathrm{~ms}$ & $12 \mu \mathrm{s}$ & 12 & ns \\
\hline 2 & $56)=32,512$ & $150 \mathrm{~ms}$ & $1.5 \mathrm{~ms}$ & 1.5 & $\mu \mathrm{s}$ \\
\hline 3 & ) $=2,763,520$ & $12.7 \mathrm{~s}$ & $127 \mathrm{~ms}$ & 127 & $\mu \mathrm{s}$ \\
\hline 4 & $\left(\begin{array}{c}256 \\
4\end{array}\right)=174,792,640$ & $13 \mathrm{~min}$ & $8 \mathrm{~s}$ & 8 & $\mathrm{~ms}$ \\
\hline 5 & $\left(\begin{array}{c}256 \\
5\end{array}\right)=8.910^{9}$ & $11 \mathrm{hr}$ & $6.7 \mathrm{~min}$ & 400 & $\mathrm{~ms}$ \\
\hline 6 & $\left(\begin{array}{c}256 \\
6\end{array}\right)=3.610^{11}$ & 20 day & $4.7 \mathrm{hr}$ & 17 & $\mathrm{~s}$ \\
\hline 7 & $\left(\begin{array}{c}256 \\
7\end{array}\right)=1.310^{13}$ & 1.9 year & 7 day & 10 & $\min$ \\
\hline 8 & $\left(\begin{array}{c}256 \\
8\end{array}\right)=4.010^{14}$ & 57 year & 7 month & 5 & $\mathrm{hr}$ \\
\hline
\end{tabular}

Figure 4: Matching time for 256-bit keys.

\begin{tabular}{|c|c|c|c|c|c|c|}
\hline \multirow{2}{*}{$\begin{array}{c}\text { Hamming " } \alpha \text { " } \\
0\end{array}$} & \multirow{2}{*}{$\begin{array}{c}\text { Numbers of } \\
\text { 128-bit streams } \\
\left(\begin{array}{c}128 \\
0\end{array}\right)=1\end{array}$} & \multirow{2}{*}{$\begin{array}{c}\begin{array}{c}\text { Matching } \\
\text { time PC }\end{array} \\
5 \mu \mathrm{s} \\
\end{array}$} & \multicolumn{2}{|c|}{$\begin{array}{l}\text { Matching } \\
\text { time server }\end{array}$} & \multicolumn{2}{|c|}{$\begin{array}{l}\text { Matching } \\
\text { time HPC }\end{array}$} \\
\hline & & & 50 & ns & 0.05 & ns \\
\hline 1 & $\left(\begin{array}{c}128 \\
1\end{array}\right)=128$ & $600 \mu \mathrm{s}$ & 6 & $\mu \mathrm{s}$ & 6 & ns \\
\hline 2 & $8)=8,128$ & $40 \mathrm{~ms}$ & 400 & $\mu \mathrm{s}$ & 400 & ns \\
\hline 3 & $\left(\begin{array}{c}128 \\
3\end{array}\right)=341,376$ & $1.7 \mathrm{~s}$ & 17 & $\mathrm{~ms}$ & 17 & $\mu s$ \\
\hline 4 & $\left(\begin{array}{c}128 \\
4\end{array}\right)=10,668,000$ & $50 \mathrm{~s}$ & 500 & $\mathrm{~ms}$ & 500 & $\mu \mathrm{s}$ \\
\hline 5 & $\left(\begin{array}{c}128 \\
5\end{array}\right)=2.610^{8}$ & $20 \mathrm{~min}$ & 12 & s & 12 & $\mathrm{~ms}$ \\
\hline 6 & $\left(\begin{array}{c}128 \\
6\end{array}\right)=5.410^{9}$ & $6.7 \mathrm{hr}$ & 4 & $\min$ & 246 & $\mathrm{~ms}$ \\
\hline 7 & $\left(\begin{array}{c}128 \\
7\end{array}\right)=9.510^{10}$ & 5 day & 1.2 & $\mathrm{hr}$ & 4.3 & s \\
\hline 8 & $\left(\begin{array}{c}128 \\
8\end{array}\right)=1.410^{12}$ & 2.2 month & 18 & $\mathrm{hr}$ & 1.1 & $\min$ \\
\hline 9 & $\left(\begin{array}{c}128 \\
9\end{array}\right)=1.910^{13}$ & 2.8 year & 10 & day & 15 & $\min$ \\
\hline 10 & $\left(\begin{array}{c}128 \\
10\end{array}\right)=2.310^{14}$ & 34 year & & month & 3 & $\mathrm{hr}$ \\
\hline
\end{tabular}

Figure 5: Matching time for 128-bit keys.

For similar error rates, the average Hamming distance " $a$ " between challenge and response is half, because half of the sub-keys are error free.

[For example if the average error rate is 3.12\%, $\boldsymbol{a}=8$ for 256-bit key, and $\boldsymbol{a}=4$ for 128-bit key]

The matching time for the same " $a$ " is smaller for 128-bit sub-keys compared with 256-bit keys.

[With a PC, and $\boldsymbol{a}=5$, it takes 11 hours to match a 256-bit key, and 20 min for a 128-bit key]

The time needed to validate " $a$ " errors of a 256 -bit key is proportional to:

$$
\mathbf{T}_{256}=\tau_{\mathrm{o}}\left(\begin{array}{c}
256 \\
a
\end{array}\right) \text {. }
$$

The time needed to validate " $a / 2$ " errors of a 128-bit key, which has the same error rate as " $\boldsymbol{a}$ " errors for a 256-bit key, is proportional to (when $\boldsymbol{a}$ is even):

$$
\mathbf{T}_{128}=\tau_{\mathrm{o}}\left(\begin{array}{l}
128 \\
a / 2
\end{array}\right) \text {. }
$$

The ratio between $\mathbf{T}_{\mathbf{2 5 6}}$, and $2 \mathbf{T}_{\mathbf{1 2 8}}$ is:

$$
\begin{gathered}
\mathbf{T}_{\mathbf{2 5 6}} / \mathbf{2} \mathbf{T}_{\mathbf{1 2 8}}=\left(\begin{array}{c}
\mathbf{2 5 6} \boldsymbol{a}
\end{array}\right) / \mathbf{2}\left(\begin{array}{c}
\mathbf{1 2 8} \\
\boldsymbol{a} / \mathbf{2}
\end{array}\right)=[256 !(128-\boldsymbol{a} / 2) !(\boldsymbol{a} / 2) !] /[2 \boldsymbol{a} !(256-\boldsymbol{a}) ! 128 !] \\
\text { [For example if } \left.\boldsymbol{a}=8: \mathbf{T}_{\mathbf{2 5 6}} / 2 \mathbf{T}_{\mathbf{1 2 8}}=\left(\begin{array}{c}
\mathbf{2 5 6} \\
\mathbf{8}
\end{array}\right) / \mathbf{2}\left(\begin{array}{c}
\mathbf{1 2 8} \\
\mathbf{4}
\end{array}\right)=256 ! 124 ! 4 ! / 28 !(248) !(128) !=\mathbf{1 . 9} \mathbf{1 0}^{7}\right]
\end{gathered}
$$

At higher error rates the efficiency of the fragmentation is enhanced, therefore higher level of noise can be added to protect IoTs with the unequally powered cryptographic scheme. 


\section{Cambou}

\subsubsection{Modelling the fragmentation in four 64-bit sub-keys of the 256-bit keys}

The matching times at various error rates " $a$ " of a model of the fragmentation in four 64-bit long sub-keys are summarized in Fig.6. the RBC with 4-way fragmentation is faster than the fragmentation in two 128bit sub-keys, which is faster than the non-fragmented scheme.

$>$ For similar error rates, the hamming distance " $a$ " between challenge and response is one fourth.

[For example if the average error rate is $3.12 \%, \boldsymbol{a}=8$ for 256-bit key, and $\boldsymbol{a}=2$ for 64-bit key]

$>$ The matching time for the same " $a$ " is smaller for 64-bit keys compared with 256-bit keys.

[With an HPC, and $\boldsymbol{a}=8$, it takes five hours to validate a 256-bit key, and 200ms to validate a 64-bit key]

$>$ The time needed to validate " $\boldsymbol{a} / \mathbf{4}$ " errors of a 64-bit key, which has the same error rate as " $\boldsymbol{a}$ " errors for a 256-bit key, is proportional to:

$$
\mathbf{T}_{64}=\tau_{\mathrm{o}}\left(\begin{array}{c}
64 \\
\boldsymbol{a} / 4
\end{array}\right)
$$

The ratio between $\mathbf{T}_{\mathbf{2 5 6}}$, and $4 \mathbf{T}_{\mathbf{6 4}}$ is:

$$
\mathbf{T}_{256} / \mathbf{4} \mathbf{T}_{64}=\left(\begin{array}{c}
\mathbf{2 5 6} \\
\boldsymbol{a}
\end{array}\right) / 2\left(\begin{array}{c}
\mathbf{6 4} \\
\boldsymbol{a} / \mathbf{4}
\end{array}\right)=[256 !(64-\boldsymbol{a} / 4) !(\boldsymbol{a} / 4) !] /[4 \boldsymbol{a} !(256-\boldsymbol{a}) ! 64 !] .
$$

\begin{tabular}{|c|c|c|c|c|c|}
\hline Hamming " $a$ " & $\begin{array}{c}\text { Numbers of } \\
64-b i t \text { streams }\end{array}$ & $\begin{array}{c}\text { Matching } \\
\text { time PC }\end{array}$ & \begin{tabular}{|c|} 
Matching \\
time server
\end{tabular} & $\begin{array}{l}\text { Matc } \\
\text { time }\end{array}$ & $\begin{array}{l}\text { hing } \\
\text { HPC }\end{array}$ \\
\hline 0 & $\left(\begin{array}{c}64 \\
0\end{array}\right)=1$ & $5 \mu \mathrm{s}$ & $50 \mathrm{~ns}$ & 0.05 & $\overline{n s}$ \\
\hline 1 & $\left(\begin{array}{c}64 \\
1\end{array}\right)=64$ & $300 \mu \mathrm{s}$ & $3 \mu \mathrm{s}$ & 3 & ns \\
\hline 2 & $\left(\begin{array}{c}64 \\
2\end{array}\right)=2,016$ & $9.5 \mathrm{~ms}$ & $95 \mu \mathrm{s}$ & 95 & ns \\
\hline 3 & $\left(\begin{array}{c}64 \\
3\end{array}\right)=62,496$ & $200 \mathrm{~ms}$ & $2 \mathrm{~ms}$ & 2 & $\mu \mathrm{s}$ \\
\hline 4 & $\left(\begin{array}{c}64 \\
4\end{array}\right)=953,064$ & $3 \mathrm{~s}$ & $30 \mathrm{~ms}$ & 30 & $\mu \mathrm{s}$ \\
\hline 5 & $\left(\begin{array}{c}64 \\
5\end{array}\right)=1.110^{7}$ & $36 \mathrm{~s}$ & $360 \mathrm{~ms}$ & 360 & $\mu s$ \\
\hline 6 & $\left(\begin{array}{c}64 \\
6\end{array}\right)=1.110^{8}$ & $5.8 \mathrm{~min}$ & $3.5 \mathrm{~s}$ & 3.5 & $\mathrm{~ms}$ \\
\hline 7 & $\left(\begin{array}{c}64 \\
7\end{array}\right)=9.310^{8}$ & $48 \mathrm{~min}$ & $29 \mathrm{~s}$ & 29 & $\mathrm{~ms}$ \\
\hline 8 & $\left(\begin{array}{c}64 \\
8\end{array}\right)=6.610^{9}$ & $5.6 \mathrm{hr}$ & $3.4 \mathrm{~min}$ & 207 & $\mathrm{~ms}$ \\
\hline 9 & $\left(\begin{array}{c}64 \\
9\end{array}\right)=4.110^{10}$ & 1.4 day & $20 \min$ & 1.2 & s \\
\hline 10 & $\left(\begin{array}{l}64 \\
1 n\end{array}\right)=2.310^{11}$ & 7.5 day & $1.8 \mathrm{hr}$ & 6.6 & s \\
\hline 11 & $\left(\begin{array}{l}64 \\
11\end{array}\right)=1.11^{12}$ & 1.2 month & $8.8 \mathrm{hr}$ & 32 & s \\
\hline 12 & $\left(\begin{array}{l}64 \\
12\end{array}\right)=4.910^{12}$ & 5.6 month & 1.7 day & 2.4 & $\min$ \\
\hline 13 & $\left(\begin{array}{l}64 \\
13\end{array}\right)=2.010^{13}$ & 1.8 year & 6.7 day & 9.6 & $\min$ \\
\hline 14 & $\left(\begin{array}{l}64 \\
14\end{array}\right)=7.210^{13}$ & 6.6 year & 24 day & 35 & $\min$ \\
\hline 15 & $\left(\begin{array}{l}64 \\
15\end{array}\right)=2.910^{14}$ & 22.7 year & 1.8 month & 2 & $\mathrm{hr}$ \\
\hline
\end{tabular}

[For example if a=8: $\quad \mathbf{T}_{\mathbf{2 5 6}} / 4 \mathbf{T}_{\mathbf{6 4}}=\left(\begin{array}{c}\mathbf{2 5 6} \\ \mathbf{8}\end{array}\right) / \mathbf{4}\left(\begin{array}{c}\mathbf{6 4} \\ \mathbf{2}\end{array}\right)=256 ! 62 ! 2 / 48$ ! (248)! (64)! $=\mathbf{5 . 1} \mathbf{1 0}^{\mathbf{1 0}}$ ]

Figure 6: Effect of noise injection on the matching time of the RBC engine for 64-bit keys.

The model suggests that the injection of much higher levels of noise is practical when a key has been fragmented into sub-keys. For example, the injection of 40 erratic bits, which represents $15.625 \%$ of a $256-$ bit key, can be matched in only 4 x 6.6 seconds with an HPC when fragmented in 64-bit chunks. The modification of the model presented above, can be generalized with a fragmentation in sub-keys of smaller size such as eight 32-bit, 16 16-bit or 32 8-bit. The model also suggest that the fragmentation can be used as a way to modulate the computing power asymmetry needed between server and IoT devices: as part of the handshake, the server can command a reduction, or an increase of the fragmentation in sub-keys. In hostile environments, the noise injection can be stronger, concurrently with higher sub-key fragmentation. The client device can unilaterally increase the fragmentation to allow the use of stronger noise injections.

\section{EXPERIMENTAL ANALYSIS}

In order to experimentally assess this protocol, a prototype was built, using SRAM PUF, window10 PC with Intel I-7 processor, and AES cryptography. 


\section{Cambou}

\subsection{Uncorrected protocol.}

Following the key exchange scheme described in section 2, a new handshake is here initiated every second to randomly generate 256-bit keys out of 256,000 cells; the keys generated by the server are based on the reading of the look up table that was downloaded during enrollment; the keys generated by the client device are based on fresh readings of the SRAM PUF, which contain errors. The results of the generation of 30 successive keys is shown in Fig.7, the keys generated by the server are on the left, the keys generated by the PUF are on the right followed by the error count and ability to match the two with the RBC engine. The keys generated by the PUF contain 4 to 16 errors, and the RBC engine always failed to match the corresponding keys because the $\mathrm{PC}$ is not powerful enough.

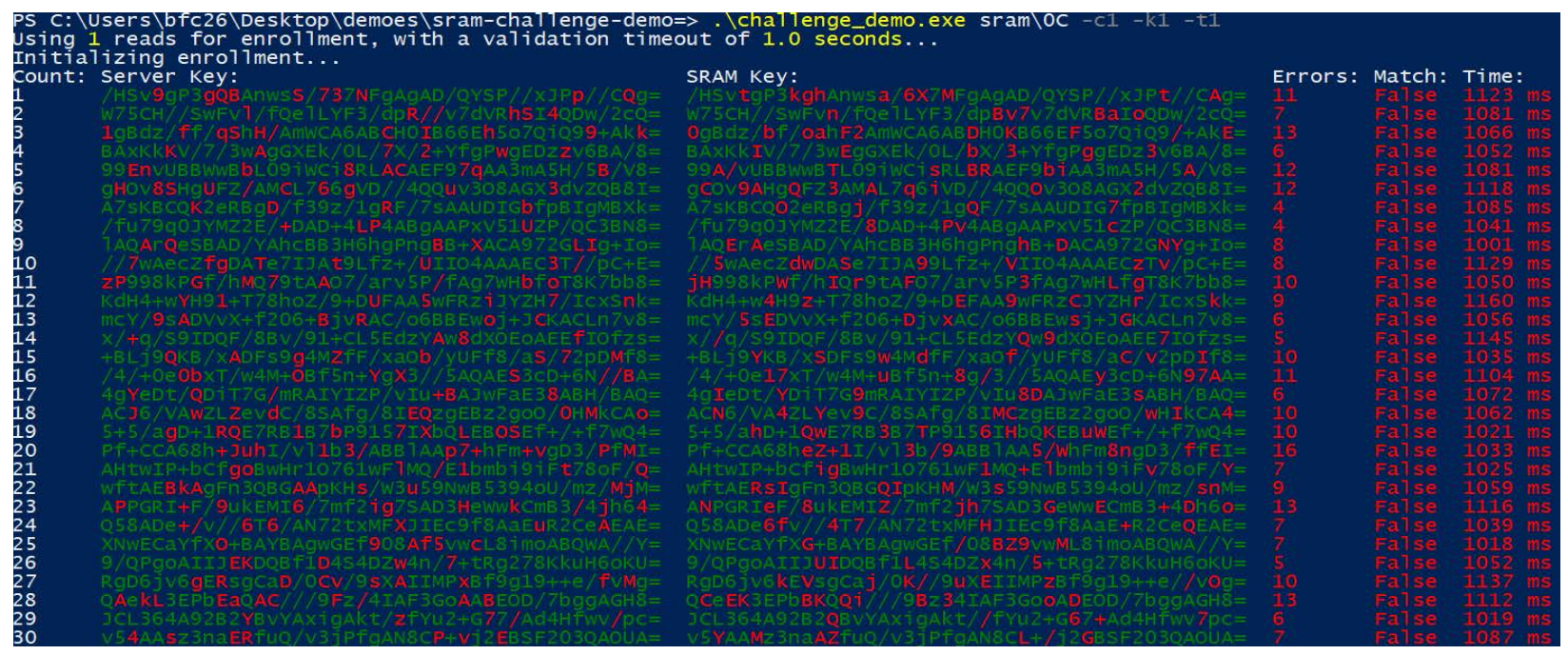

Figure 7: Random key exchange between the server and a non-corrected SRAM PUF.

\subsection{Use of ternary states}

The value of using PUFs with three states was demonstrated with SRAM PUFs. One thousand power-offpower-on cycles during enrollment allow the identification of the cells with erratic behavior. The protocol is the same one as the one described section 2, but the fuzzy cells are eliminated to reduce the error rates. The result of 30 successive cycles is shown in Fig.8. The error rates observed are extremely low; only two errors were observed after one thousand handshakes, out of 256,000 bits, an error rate of $10^{-5}$. The RBC engine was indeed successful to match the two keys, and was proven effective in the rare case of a one-bit mismatch. The estimated average latency of the RBC engine with AES is $300 \mathrm{~ms}$.

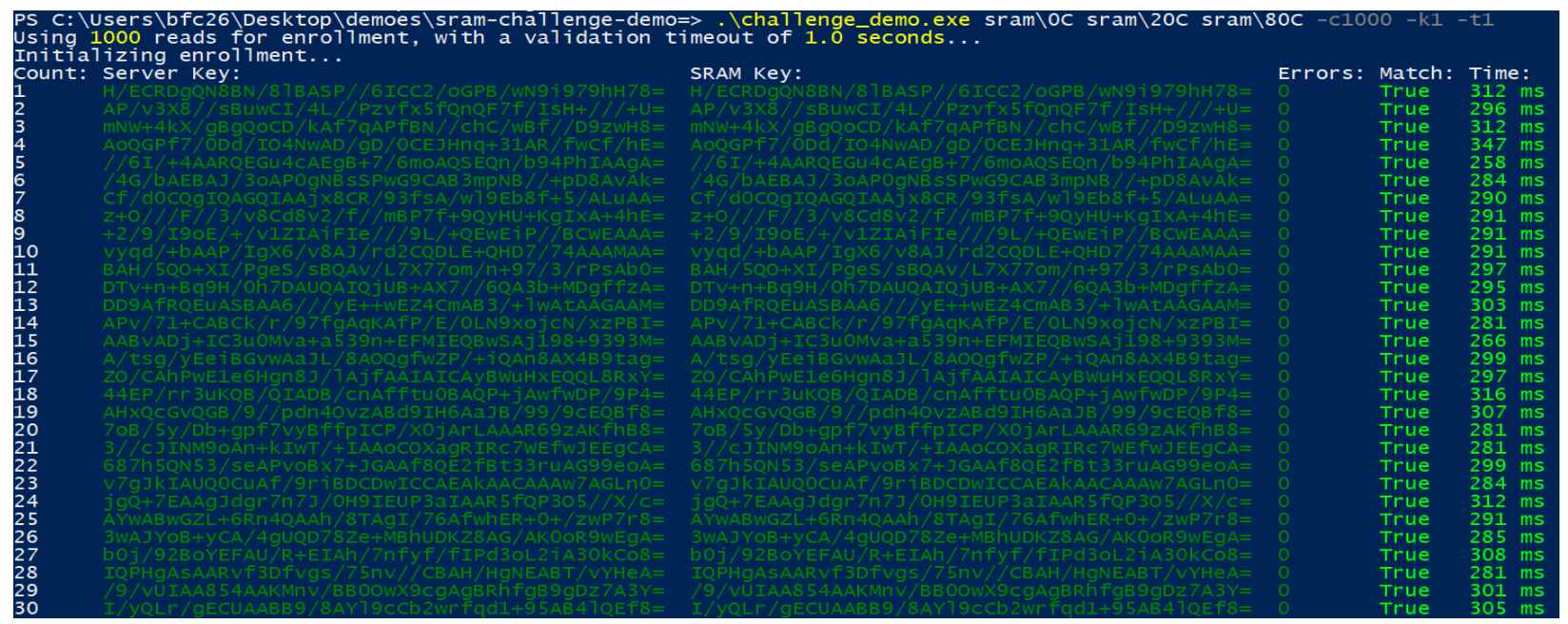


Figure 8: Random key exchange between the server and a ternary SRAM PUF.

\subsection{Use of heavy key fragmentation}

The efficiency of the key fragmentation, was demonstrated with the RBC protocol as applied to uncorrected PUFs. The result of 30 successive cycles is presented in Fig. 9 with a fragmentation by 16 16-bit sub-keys. The latency of such protocol is still in the $300 \mathrm{~ms}$ range, and all keys were matched by the RBC engine. The $\mathrm{PC}$ is powerful enough to handle the protocol, which is consistent with the model developed in section 3. This demonstrates that error-less communication can be established between a PC and a low power IoT with SRAM PUF while either using ternary states to reduce the error rates, or by the use of the RBC with heavy fragmentation. When the fragmentation is reduced to a factor 8 or less the method is not working as well, unless it is combined with a ternary PUF with lower error rates.

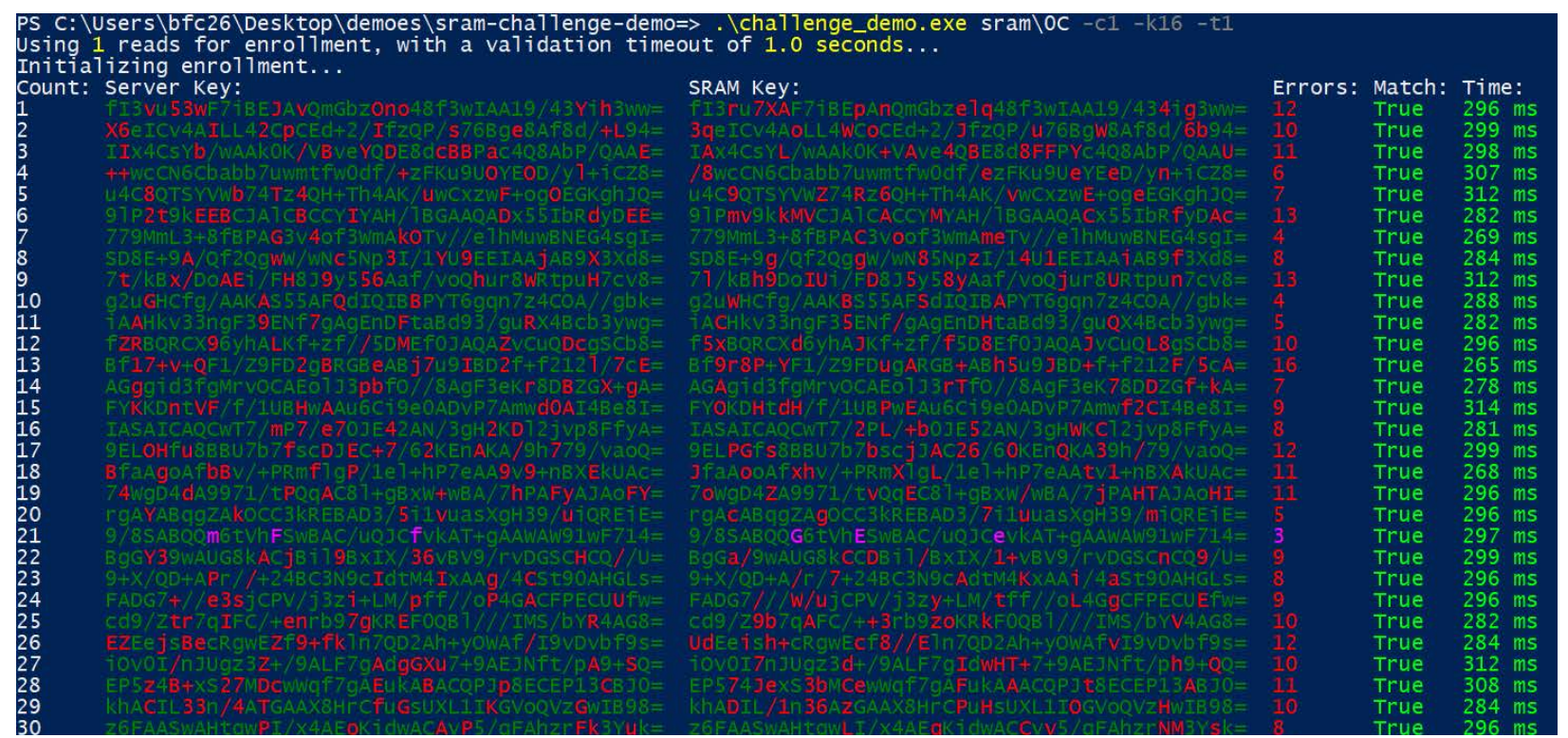

Figure 9: Random key exchange between the server and a PUF with fragmentation by 16.

\subsection{Use of lighter key fragmentation}

The use of a lighter key fragmentation shows the potential of unequally powered cryptography. In the experiment shown in Fig.10, the result of 30 successive readings is shown with a fragmentation by four 64bit long sub-keys. The RBC engine of the PC was given 90 second to complete the matching cycle. Only three events resulted in a match successful in less than $400 \mathrm{~ms}$, however the error rates were only four or five corresponding to a Hamming distance close to one for the 64-bit long sub-keys. All other cases are showing a longer period of time to find a match, one case was above the 90s limit. A more detailed analysis shows that in 12 events, at least one sub-key has 5 errors; the average latency, as predicted by the model of section 3.32 is 30 seconds, which is in line with the experimental results. It is also noticeable that in 8 events, at least one sub-key has 3 errors; the average latency predicted by the model of section 3.32 is 3 seconds, also in line with the experimental results. The tool presented in this section 4 was used over an extended period of time to validate the models developed in section 3 . 


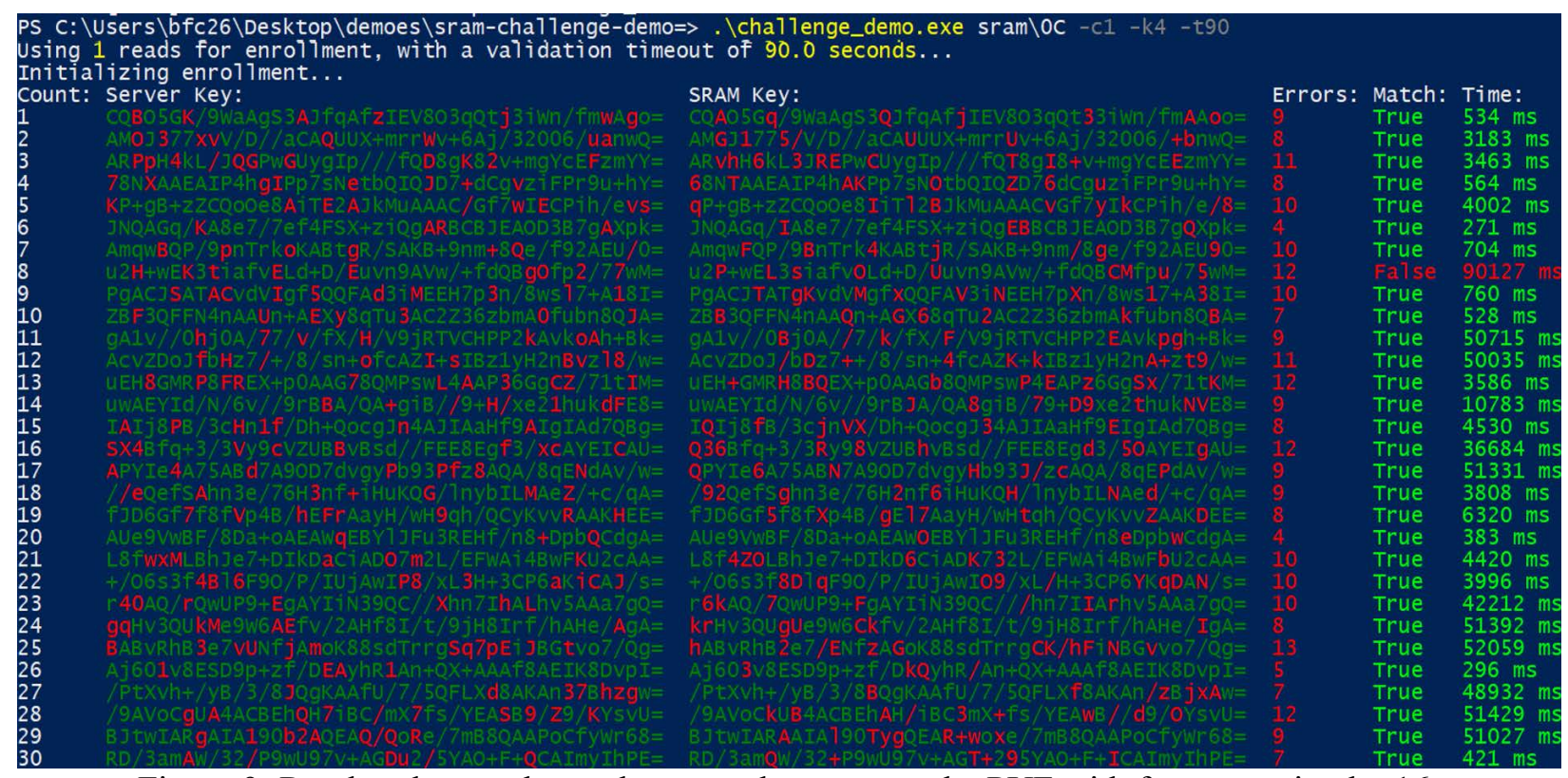

Figure 9: Random key exchange between the server and a PUF with fragmentation by 16.

\section{EFFICIENCY OF HIGH PERFORMANCE COMPUTERS}

A server 1,000 more powerful than the PC would have been able to establish successful matchings in all 30 readings of section 4.4 in less than $100 \mathrm{~ms}$, while none can be successful with a PC. The objective of this section is to predict the efficiency of HPC at various levels of noise, and key fragmentations. The summary of the modelling work is presented Fig. 10. The left column shows the error rate in \% for 256-bit keys, and 64-bit sub-keys. The second column shows the failure rates when the RBC is correcting hamming distances of " $a$ " or below. The three columns in the right show the number of successive queries needed to get false reject rates (FRRs) below $0.1 \%$, then the latency needed when either HPCs or regular PCs are used on the server side. In order to be able to validate the key exchange with a low FRR, the highest acceptable error rate is only $0.3 \%$ with 256 -bit keys. The acceptable error rate jumps to $7 \%$ with a 64 -bit fragmentation. In Fig.8, two matching algorithms are compared, one with one handshake (1Q), and the one with two handshakes (2Q). It is statistically faster to trigger a second handshake if the latency of the first one is too slow, because the Hamming distance is small in most cases. However, the second query is not always desirable from a security stand point.

The error rates reported in this analysis are due to the combination of the naturally generated errors from the PUFs, and the ones injected separately. The algorithm based on 256-bit fragmentation cannot handle error rates higher than $0.3 \%$ without prohibitive false rejection rates (FRRs). The algorithm based on a $64-$ bit fragmentation can respond to error rates as high as 7\%, which is desirable to enhance the protection of the terminal devices, with low FRR. It is therefore possible to use the method with non-corrected PUFs and with natural error rates in the 3\% range. In our research work, we also analyzed the impact of 32-bit fragmentations, which allows the use of even more erratic PUFs. The use of higher levels of fragmentation, for example 16-bits and 8-bit fragmentations, is not recommended because this increases the high false acceptance rates (FAR), i.e. the adversary could successfully generate false keys. 


\begin{tabular}{|c|c|c|c|c|c|c|c|c|c|c|c|c|c|c|c|c|}
\hline \multirow{2}{*}{$\begin{array}{c}\text { 256-bit } \\
\text { PUF CRP } \\
\text { error rate } \\
\% \\
\end{array}$} & \multicolumn{6}{|c|}{$\begin{array}{c}\text { Failure rate (FRR) in } \% \\
\text { When matching responses } \\
\text { with Hamming distances " } a \text { " }\end{array}$} & \multicolumn{6}{|c|}{$\begin{array}{l}\text { Queries needed } \\
\text { to get FRR }<0.1 \%\end{array}$} & \multicolumn{2}{|c|}{$\begin{array}{l}\text { Matching } \\
\text { Latency for } \\
\text { HPC }\end{array}$} & \multicolumn{2}{|c|}{$\begin{array}{l}\text { Matching } \\
\text { Latency for } \\
\text { PC }\end{array}$} \\
\hline & $a=1$ & $a=2$ & $a=3$ & & $a=4$ & $a=5$ & $a=1$ & $a=2$ & & $a=3$ & $a=4$ & $a=5$ & 1Q & $2 Q$ & 1Q & $2 Q$ \\
\hline & 99 & 98 & 95 & 5 & 88 & 78 & NA & NA & & NA & NA & NA & NA & NA & NA & NA \\
\hline & 72 & 47 & 26 & & 12 & 4.6 & NA & NA & & NA & 4 & 3 & NA & NA & NA & NA \\
\hline & 18 & 4.3 & 0.8 & 8 & 0.12 & 0.015 & 4 & 3 & & 2 & 2 & 1 & $400 \mathrm{~ms}$ & $250 \mu \mathrm{s}$ & $11 \mathrm{hr}$ & $25 s$ \\
\hline 0.1 & 2.8 & 0.23 & \begin{tabular}{l|l|}
3 & 0.01 \\
\end{tabular} & & $0.01<$ & $0.01<$ & 2 & 2 & & 2 & 1 & 1 & $8 \mathrm{~ms}$ & $12 \mathrm{~ns}<$ & $13 \mathrm{~min}$ & $1 \mathrm{~ms}<$ \\
\hline 0.03 & 0.28 & 0.01 & $\begin{array}{ll}<< & 0.01 \\
\end{array}$ & \begin{tabular}{l|l}
$1<$ & 0 \\
\end{tabular} & $0.01<$ & $0.01<$ & 2 & 1 & & 1 & 1 & 1 & $1.5 \mu \mathrm{s}$ & $12 n s<$ & $150 \mathrm{~ms}$ & $1 \mathrm{~ms}<$ \\
\hline 0.01 & 0.03 & 0.01 & \begin{tabular}{l|l|}
$<$ & 0.01 \\
\end{tabular} & & $0.01<$ & $0.01<$ & 1 & 1 & & 1 & 1 & 1 & 12 ns $<$ & - & $1 \mathrm{~ms}<$ & - \\
\hline \multirow{2}{*}{ 64-bit } & \multicolumn{6}{|c|}{ Failure rate (FRR) in \% } & \multicolumn{6}{|c|}{ Queries needed } & \multicolumn{2}{|c|}{ HPC } & \multicolumn{2}{|c|}{ PC } \\
\hline & $a=5$ & $a=6$ & $a=7$ & $a=8$ & $a=9$ & $a=10$ & $a=5$ & $a=6$ & $a=7$ & $a=8$ & $a=9$ & $a=10$ & 1Q & $2 Q$ & $1 Q$ & $2 Q$ \\
\hline 4 & 61 & 46 & 31 & 20 & 11 & 6 & NA & NA & NA & NA & 4 & 3 & NA & NA & NA & NA \\
\hline & 29 & 16.5 & 8.5 & 4 & 1.7 & 0.6 & NA & 4 & 3 & 3 & 2 & 2 & NA & $2.4 \mathrm{~s}$ & NA & 1.4day \\
\hline 5 & 10.5 & 4.5 & 1.7 & 0.6 & 0.2 & 0.05 & 4 & 3 & 2 & 2 & 2 & 1 & $7 s$ & $60 \mathrm{~ms}$ & 7.5day & $1.5 \mathrm{hr}$ \\
\hline 3 & 1.4 & 0.4 & 0.08 & 0.02 & $0.01<$ & $0.01<$ & 2 & 2 & 1 & 1 & 1 & 1 & $29 \mathrm{~ms}$ & $1 \mathrm{~ms}$ & $48 \mathrm{~min}$ & $1 \mathrm{~min}$ \\
\hline 1 & $0.01<$ & $0.01<$ & $0.01<$ & $0.01<$ & $0.01<$ & $0.01<$ & 1 & 1 & 1 & 1 & 1 & 1 & $0.3 \mathrm{~ms}<$ & - & $30 \mathrm{~s}$ & - \\
\hline 0.3 & $0.01<$ & $0.01<$ & $0.01<$ & $0.01<$ & $0.01<$ & $0.01<$ & 1 & 1 & 1 & 1 & 1 & 1 & $0.3 \mathrm{~ms}<$ & - & $30 \mathrm{~s}$ & - \\
\hline
\end{tabular}

Figure 8: Analysis of the protocol with SRAM-PUFs. Latency of the key matching algorithm at various levels of error rates for FRR $<0.1 \%$.Comparison between 256-bit keys, and 64-bit sub-keys. When the matching is too long after a first handshake (1Q), a second handshake (2Q) results in lower latencies.

\section{CONCLUSION}

The experimental work presented in this paper was conducted with SRAM PUFs, Window-10 PCs with Intel I-7 processor, while the efficiencies of high performance computers (HPCs) were modelled. The models were validated by varying the noise level injected (from $10^{-5}$ to about 5\%) into 256-bit long cryptographic keys, and encrypting authentication messages with the advanced encryption standard (AES). The noise level was adjusted by selectively masking the instable cells of the SRAM PUFs. At low noise levels, the computing power of the single-core PC is appropriate to independently find the matching keys. The fragmentation of the keys into smaller blocks, that generate sub-keys, allows the injection of much higher levels of noise, and enhances the value of the proposed schemes. For example, with a fragmentation by 4 of 256-bit long keys, and with a 5\% noise injection, the HPC is modelled to find the matching keys with two handshakes in only $60 \mathrm{~ms}$, versus 1.5 hours with the single-core PC; when the IoTs are set to reject a server not able to respond within one second, only HPCs can communicate with the IoT. We are currently designing experiments to validate these modelling results with NAU's 1,600-core HPC, and with various PUFs that are based on different components such as memristors.

The generalization of the methods to secure public key infrastructures (PKIs) is also under consideration. Rather than encrypting with AES an authentication message using the private key generated from the PUF, as presented in this paper, the client device will generate public keys that are openly shared with the PKI network, see Fig.9. The server, with the RBC engine, independently validates the public keys, and posts it in a public ledger. The noise is injected to create a mismatch between the keys used by the client device, and the ones generated by the ternary PUF; only servers equipped with powerful computing power can validate these keys.

\section{ACKNOWLEDGMENTS}

The author acknowledge the outstanding contribution of the graduate, and undergraduate students working in the cybersecurity laboratory of Northern Arizona University. Christopher Philabaum, Duane Booher, and Sareh Assiri developed several software implementations of this architecture. Vince Rodriguez, and Ian Burke designed and characterized the electronic board driving the SRAM PUFs that were used in the 
experimental section of this paper. Christopher Coffey, the Engineer in charge of NAU's HPC provided highly valuable insights in the architecture. And, Cristy Salanga, and Dr. William Aylor from the research and innovation organization provided extraordinary support.

\section{REFERENCES}

Becker, G. T., A. Wild and T. Güneysu. 2015. "Security analysis of index-based syndrome coding for PUFbased key generation". In 2015 IEEE International Symposium on Hardware Oriented Security and Trust (HOST), Washington, DC.

Boehm, H. M. 2010. "Error correction coding for physical unclonable functions". In Austrochip-2010, Workshop in Microelectronics.

Cambou, B., and D. Telesca. 2018. "Ternary Computing to Strengthen Information Assurance, Development of Ternary State based public key exchange'. IEEE, SAI-2018, Computing Conference, London, UK.

Cambou, B., P. Flikkema, J. Palmer, D. Telesca, and C. Philabaum. 2018. "Can Ternary Computing Improve Information Assurance?" Journal of Cryptography, MDPI-2018.

Cambou, B., C. Philabaum, D. Duane Booher, and D. Telesca. Accepted 2019. "Response-based Cryptography Methods with Physical Unclonable Functions'. Future of Information and Communication Conference, FICC-2019.

Cambou, B., and M. Orlowski. 2016. "Design of Physical Unclonable Functions with ReRAM and ternary states". Cyber and Information Security Research Conference, CISR-2016, Oak Ridge, TN, USA.

Cambou, B., F. Afghah, D. Sonderegger, J. Taggart, H. Barnaby and M. Kozicki. 2017. "Ag conductive bridge RAMs for physical unclonable functions". In 2017 IEEE International Symposium on Hardware Oriented Security and Trust (HOST), McLean, USA.

Chen, T. I. B., F. M. Willems, R. Maes, E. v. d. Sluis, and G. Selimis. 2017. "A Robust SRAM-PUF Key Generation Scheme Based on Polar Codes". In arXiv:1701.07320 [cs.IT].

Chen, A. 2015. "Comprehensive Assessment of RRAM-based PUF for Hardware Security Applications". 978-1-4673-9894-7/15/IEDM IEEE.

Delvaux, J., D. Gu, D. Schellekens and I. Verbauwhede. 2015. "Helper Data Algorithms for PUF-Based Key Generation: Overview and Analysis". IEEE Transactions on Computer-Aided Design of Integrated Circuits and Systems, vol. 34, no. 6, pp. 889-902.

Fernando, L., N.C. Wang, G.F.R. Soysa. 2018. “Transaction Device with Noise Signal Encryption”. US patent 7,392,396 B2.

Fiskle, M. 2016. "Hiding a Public Key Exchange in Noise". Patent application WO 2016/187432 A1.

Gao, Y., D. Ranasinghe, S. Al-Sarawi, O. Kavehei, and D. Abbott. 2016. "Emerging Physical Unclonable Functions with nanotechnologies". IEEE, DOI:10.1109/ACCESS.2015.2503432.

Herder, C., M. Yu, and F. Koushanfar. 2014. "Physical Unclonable Functions and Applications: A Tutorial". Proceedings of the IEEE, vol. 102, no. 8, pp. 1126-1141.

Hiller, M., M. Weiner, L. Rodrigues, M. Birkner and G. Sigl.2013. "Breaking through Fixed PUF Block Limitations with Differential Sequence Coding and Convolutional Codes". In TrustED'13.

Holcomb, D. E., W. P. Burleson, and K. Fu. 2008. "Power-up SRAM state as an Identifying Fingerprint and Source of TRN". IEEE Transaction on Computing, vol 57, No 11.

Janus, S., J. Martin, and U. Savagaonkar. 2017. "Preventing Patent Recognition in Electronic Code Book Encryption”. Patent disclosure US 2017/0070342 A1. 
Jin, Y. 2015. "Introduction to hardware security". Electronics, 4, 763-784; doi: 10.3390/electronics 4040763.

Kang, H., Y. Hori, T. Katashita, M. Hagiwara and K. Iwamura. 2014. "Cryptographie key generation from PUF data using efficient fuzzy extractors". In 16th International Conference on Advanced Communication Technology, Pyeongchang, Korea.

Korenda, A., F. Afghah and B. Cambou. 2018. "A Secret Key Generation Scheme for Internet of Things using Ternary-States ReRAM-based Physical Unclonable Functions". In International Wireless Communications and Mobile Computing Conference (IWCMC 2018).

Maes, R., P. Tuyls and I. Verbauwhede. 2009. "A Soft Decision Helper Data Algorithm for SRAM PUFs". In 2009 IEEE International Symposium on Information Theory.

Prabhu, P., A. Akel, L. M. Grupp, W-K S. Yu, G. E. Suh, E. Kan, and S. Swanson. 2011. "Extracting Device Fingerprints from Flash Memory by Exploiting Physical Variations". In 4th international conference on Trust and trustworthy computing.

Rahman, M. T., F. Rahman, D. Forte and M. Tehranipoor. 2016. "An Aging-Resistant RO-PUF for Reliable Key Generation". IEEE Trans. on Emerging Topics in Computing, vol. 4, no. 3.

Taniguchi, M., M. Shiozaki, H. Kubo and T. Fujino. 2013. "A stable key generation from PUF responses with a Fuzzy Extractor for cryptographic authentications". In IEEE 2nd Global Conference on Consumer Electronics (GCCE), Tokyo, Japan.

\section{AUTHOR BIOGRAPHY}

BERTRAND FRANCIS CAMBOU is a Professor of Practice in the School of informatics Computing and Cyber Systems (SICCS) at Northern Arizona University (NAU). He holds a Doctorate degree in Electronic Materials from the University of Paris South, and an Engineering degree in Electronics from Supelec, Paris. His research interests lie in developing end-to-end cybersecurity solutions based on Nanoelectronic materials. $\mathrm{He}$ is an Invention Ambassador of the American Association for the Advancement of Science (AAAS), and a Senior Member of the National Academy of Inventors (NAI) . His email address is: bertrand.cambou@nau.edu 